\title{
Relative finger position influences whether you can localize tactile stimuli
}

\author{
K. E. Overvliet $\cdot$ H. A. Anema $\cdot$ E. Brenner $\cdot$ \\ H. C. Dijkerman · J. B. J. Smeets
}

Received: 17 March 2010 / Accepted: 23 October 2010 / Published online: 16 November 2010

(C) The Author(s) 2010. This article is published with open access at Springerlink.com

\begin{abstract}
To investigate whether the relative positions of the fingers influence tactile localization, participants were asked to localize tactile stimuli applied to their fingertips. We measured the location and rate of errors for three finger configurations: fingers stretched out and together so that they are touching each other, fingers stretched out and spread apart maximally and fingers stretched out with the two hands on top of each other so that the fingers are interwoven. When the fingers contact each other, it is likely that the error rate to the adjacent fingers will be higher than when the fingers are spread apart. In particular, we reasoned that localization would probably improve when the fingers are spread. We aimed at assessing whether such
\end{abstract}

K. E. Overvliet $(\bowtie) \cdot$ E. Brenner · J. B. J. Smeets

Research Institute MOVE, Faculty of Human Movement Sciences,

VU University Amsterdam, Van der Boechorststraat 9,

1081 BT Amsterdam, The Netherlands

e-mail: krista.overvliet@gmail.com

K. E. Overvliet

Departament de Psicologia Bàsica, Universitat de Barcelona,

Passeig de la Vall d'Hebron, 171, 08035 Barcelona, Spain

K. E. Overvliet

Laboratory of Experimental Psychology,

University of Leuven, Tiensestraat 102,

3000 Leuven, Belgium

\section{H. A. Anema · H. C. Dijkerman}

Experimental Psychology, Helmholtz Institute,

Utrecht University, Heidelberglaan 2,

3584 CS Utrecht, The Netherlands

\section{H. A. Anema}

Department of Public Health, Amsterdam Medical Centre,

University of Amsterdam, Meibergdreef 9,

1100 DD Amsterdam, The Netherlands adjacency was measured in external coordinates (taking proprioception into account) or on the body (in skin coordinates). The results confirmed that the error rate was lower when the fingers were spread. However, there was no decrease in error rate to neighbouring fingertips in the fingers spread condition in comparison with the fingers together condition. In an additional experiment, we showed that the lower error rate when the fingers were spread was not related to the continuous tactile input from the neighbouring fingers when the fingers were together. The current results suggest that information from proprioception is taken into account in perceiving the location of a stimulus on one of the fingertips.

Keywords Touch $\cdot$ Proprioception $\cdot$ Haptics $\cdot$ Fingers . Hands · Human

\section{Introduction}

If you want to identify an object by touch, you need to combine tactile input with information about the positions of your hands and fingers. When you touch something, tactile information is initially coded in a somatotopical map of skin coordinates in area $3 \mathrm{~b}$ of the somatosensory cortex (e.g. Kaas 1983). In a later stage, in area 5, this is combined with information from proprioception that originates from underlying muscles and joints and that was initially coded in area 3a of the somatosensory cortex (e.g. Kaas 1983). This sequence has been demonstrated for the arms and legs (e.g. Azañon and Soto-Faraco 2008; Schicke and Röder 2006) but has never specifically been confirmed for the fingers. In the current study, we aimed at demonstrating tactile and proprioceptive integration by asking participants to localize tactile stimuli on the fingertips in different finger 
configurations. We hypothesize that if tactile information on the fingertips is combined with information from proprioception to obtain information about points' locations in an external reference frame, participants will make fewer errors when the fingers are spread and more errors when they are interwoven in an unusual manner.

Natsoulas and Dubanoski (1964) demonstrated that the orientation of the stimulated skin influences the final percept of a tactile stimulus. Their results show that the orientation of your head influences the perception of the orientation of a shape drawn on your forehead. More recently, in a study by Yamamoto and Kitazawa (2001), participants had to indicate the temporal order of two tactile stimuli delivered to the left and right hand at different interstimulus intervals. The hands could be in different configurations, either crossed or uncrossed. For long inter-stimulus intervals, participants performed almost perfectly irrespectively of whether the hands were crossed or uncrossed. However, when the inter-stimulus intervals were short $(<300 \mathrm{~ms})$ and hands were crossed, participants often misperceived the temporal order of the two stimuli. When the hands were uncrossed, the participants could still indicate the temporal order correctly with inter-stimulus intervals as short as $70 \mathrm{~ms}$. This suggests that for short inter-stimulus intervals, the temporal order of the stimuli was perceived as if the hands were uncrossed. This may be interpreted that the initial coding of this tactile perception is coded in local 'skin' coordinates. The longer inter-stimulus intervals allowed the temporal order to be perceived correctly, regardless of hand configuration. This suggests that the initial coding of the tactile perception is translated into external coordinates, which takes the positions of the arms into account.

Soto-Faraco et al. (2004) provide more evidence that the final tactile percept is influenced by the spatial location of the limbs. Their participants were asked to make judgements about the presence and location of a vibrotactile stimulus presented to the thumb or index finger of one hand while attempting to ignore distractor stimuli on the thumb and index finger of the other hand. They showed that when the target and distractor were on congruent digits (i.e. both on index fingers), the reaction times were shorter and error rates were lower than when they were on incongruent digits (one on an index finger and the other on a thumb). The difference between congruent and incongruent presentations was smaller when the target and distractor were further apart: the separation between the hands in external space determined how strongly the stimuli influenced each other. Apparently, the effect of distractors does depend not (only) on the stimulated skin sites but also on the distance between the stimulated body parts.

Thus, tactile perception depends on a translation of the stimulated skin sites into spatial locations of the stimuli in the external world, a process that takes time and requires information about the location of the stimulated body part in external space. So, tactile localization appears to depend on posture, the time that elapsed since tactile stimulation and as well as on the local tactile sensitivity. An interesting question is whether this only holds for the object that is touched or whether this is also the case for the representation of the fingers. The fingers of one hand are the only body parts that regularly operate in a fixed configuration within a small space. This, together with the high spatial acuity of the tactile receptors, makes fingers particularly suited for identifying and manipulating objects. Thus, the fingers are somewhat special, and some evidence suggests that there may be different representations for the location of fingers (than for other body parts) within the brain (e.g. Haggard et al. 2006).

In their study, Haggard et al. (2006) looked at the effects of various configurations of the hands and fingers in tactile processing. Participants were asked to either report whether they detected a tactile stimulus applied to the fingertips, or indicate which finger was stimulated (but not of which hand), or indicate which hand was stimulated (but not which finger). This task was performed both with the hands next to each other and with the fingers of the two hands interwoven. There was an increase in error rate when the fingers were interwoven when participants had to identify the stimulated hand (left or right), but not when they had to identify the stimulated finger (irrespective of the hand). Therefore, it was suggested that hands and fingers were processed separately and that the configuration of the hands did not have an effect on the identification of the stimulated finger. The fingers may be represented in a somatotopic skin space, while hands are represented in external space. Moreover, they concluded that identifying a finger does not necessarily imply that the hand to which it belongs is also known.

In another study of tactile finger representations, Craig (2003) asked subjects to judge the order of presentation of two moving stimuli. These stimuli were moving from one side of the finger pad to the other. He found that the direction of motion of the tactile stimulation influenced the temporal order judgement. When the direction of the movement of the first stimulus was towards the second stimulus, temporal order judgements were more accurate than when it was away from the second stimulus. He found this effect when the fingers were parallel to each other. When he increased the angle between the fingers, the difference in performance decreased. The spatial locations and orientation of the fingers were apparently influencing the performance on this temporal order judgement task, implying that, in contrast to what Haggard et al. (2006) claimed, tactile perception on the fingers is represented in an external, spatial reference frame. 
Other evidence of finger posture influencing tactile processing comes from a study by some of the current authors (Overvliet et al. 2008). In their study, participants had to detect the absence of a line segment under one of their fingertips when their fingers were in different configurations: all fingertips positioned on a single straight line which had a gap just beneath one of the fingertips, or the fingers in a relaxed posture with separate line segments under all but one fingertip. In the condition in which the fingertips were on a straight line, detection of the gap was much faster than when the fingers were in a relaxed posture (with separate line segments). Apparently, the configuration of the fingers influenced detection because the tactile input on the fingertips was identical in both conditions. It was concluded that the integration of the perceived line segments into an object was the critical component for observing the faster gap detection times. The proprioceptive information about the location of the fingers was probably essential for integrating the perceived line segments into a single percept when the fingertips were aligned and close together.

Though our fingers are very sensitive to tactile information (Weinstein 1968), the way how we determine the location of tactile stimuli on our fingers remains largely unknown. An extensive investigation into how accurate we are in localizing near-threshold tactile stimulation (von Frey hair applications) of the fingers by Schweizer et al. (2000) showed that potential stimulation points that were close to the actual stimulation point were selected more frequently than ones that were further away. Nearby stimulation points were selected even more frequently than expected on the basis of chance when they belonged to a neighbouring finger. Schweizer et al. concluded that digitoverlapping receptive fields are responsible for the errors made to neighbouring fingers. The idea of digit-overlapping receptive fields in the somatosensory cortex is far from new (Iwamura et al. 1983a, b; McKenna et al. 1982). If these overlapping receptive fields are linked to skin locations, proximity of two adjacent fingers would not change the distribution of errors. Since we observed that the (relative) positions of the hands and fingers are relevant when processing tactile information, the somatotopical receptive fields might not overlap in terms of skin location but in terms of an external coordinate system. If this is the case, enlarging the distance between the fingers should improve localization. In particular, spreading the fingers should decrease the tendency to choose the adjacent position on the neighbouring finger, because the distance is increased.

To test this hypothesis, we used a variation on the paradigm used by Schweizer et al. (2000). We studied the errors that participants made when localizing a near-threshold stimulus presented to one of thirty locations at the fingertips. We used three different finger configurations: fingers together, fingers spread and fingers interwoven. If the fingers are represented somatotopically, participants will make the same errors to the neighbouring finger in 'fingers spread', 'fingers together' and 'fingers interwoven' conditions. However, if they are represented spatially, the errors will be distributed differently for each finger configuration.

\section{Experiment 1}

Method

\section{Participants}

Ten participants, two men and eight women, with an average age of 27.5 years (range 20-38) participated in this experiment. The participants were undergraduate students and co-workers. None of the participants had known problems with tactile perception due to, for example, numbness, callus or scar tissue on any of their fingers. We did not consider handedness to be an important factor in the current study. All participants signed an informed consent form before participating in the experiment. The study was part of a programme that has been approved by the ethical committee of the Faculty of Human Movement Sciences of the VU University.

\section{Stimuli and set-up}

We used a point localization test. The stimulus that was used was a von Frey hair of $0.07 \mathrm{~g}$ (North Coast Medical, Touch-Test, Sensory Evaluator Size 2.83). We chose this size because earlier findings by other experimenters indicated that this generated an error rate of about $50 \%$ (Schweizer et al. 2000, 2001), which is the optimal rate for detecting differences in performance between the conditions. Three finger configurations were used: fingers stretched out and together so that they are touching each other, fingers stretched out and spread apart maximally and fingers stretched out with the two hands on top of each other so that the fingers are interwoven (Fig. 1a-c). We measured the size of the fingertip by pressing it onto a ruler. We took the width of the fingertip at the location of the bump in the centre of each finger pad. We then marked 3 evenly distributed and aligned dots at that location on each finger pad of both hands of the participant, at $0.25,0.5$ and 0.75 of the total finger width. Thus, the distances between the dots depended on finger width and were not absolutely spaced. A screen with a curtain covering the opening through which the participant extended his or her arms prevented the participant from seeing the hands. A map of the two hands with the numbered positions (Fig. 1d) was attached to the screen in such a way that the participant 

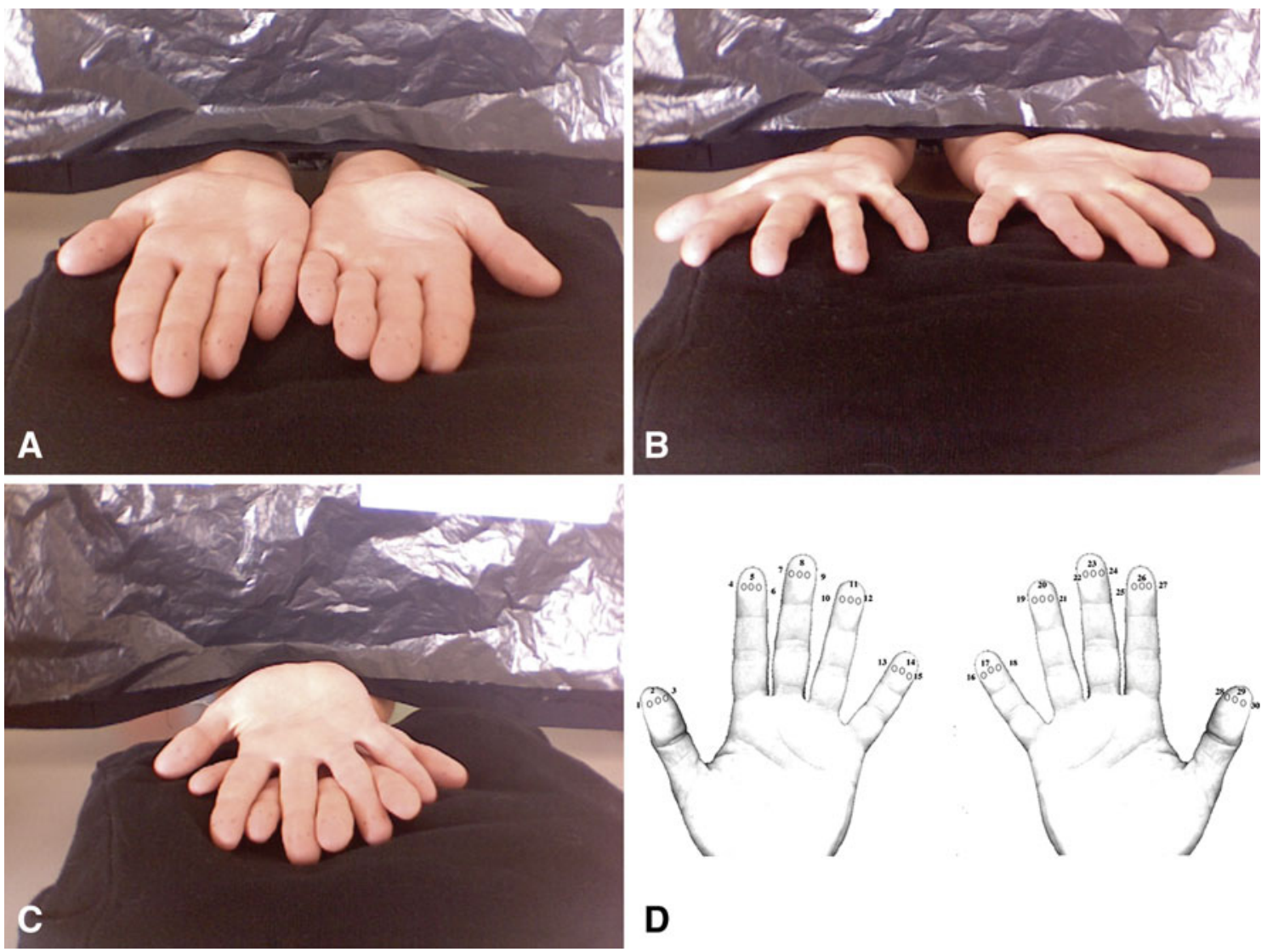

Fig. 1 The different finger configurations used in experiment 1: a fingers together, $\mathbf{b}$ fingers spread and $\mathbf{c}$ fingers interwoven. $\mathbf{d}$ The map of the two hands that was shown to our participants, with numbered dots indicating the possible stimulation sites

could easily read it. We kept this response map the same in all three experimental conditions; this might cause an overall increase in response time in the interwoven condition (if a spatial reference frame is used), but it should not affect error rates.

There were 150 trials for every finger configuration: 5 repetitions for each of the 30 ( 3 dots $\times 5$ fingers $\times 2$ hands) possible stimulation locations. The three finger configurations were measured in different blocks, randomized in order across participants. Within a block, the 150 trials were presented in a random order.

\section{Procedure}

After the experimental procedure was explained to the participant and the informed consent form was signed, participants were seated behind the screen. They were asked to stretch out their arms through the opening in the screen and place their hands with their palms up on a pillow, assuming one of the three finger configurations (Fig. 1a-c). A warning signal indicated that the stimulus was going to be applied, after which the stimulus was applied at one of the thirty marked locations. The location was tactually stimulated with the von Frey hair applying just enough force for the hair to start bending (which indicates that the maximum application force of the von Frey hair is reached). The von Frey hair application was done manually. The stimulus was applied once, continuously for one second. It sometimes happened that the von Frey hair slipped off of the desired location. If it did so, the trial was repeated at the end of the block of trials. The participant's task was to name the location, as indicated by the numbers on the map, at which they felt the stimulus. They were allowed to take as much time as necessary. However, if they did not feel the stimulus and spent too much time thinking (more than about $20 \mathrm{~s}$ ), they were encouraged to guess. The participant had to name a location, even if they had not felt the stimulus. The experimenter entered the named location in the computer, and the next trial was started.

\section{Results}

\section{Correct responses}

Overall task performance per condition was calculated by determining the proportion of correct responses for each participant. The average values were $0.44 \pm 0.04$, $0.56 \pm 0.05$ and $0.40 \pm 0.05$ (mean \pm standard error) for 
fingers together, finger spread and fingers interwoven, respectively. Schweizer et al. (2000) found a proportion of $48 \%$ correct trials, which is between our values for fingers together and fingers spread. In their study, the positions of the fingers with respect to each other were not experimentally controlled.

We found a main effect of finger configuration on the proportions correct (repeated-measures ANOVA; $F(2.18)=$ 26.44, $P<0.001$ ). Paired-samples $t$ tests (Bonferroni-corrected) revealed that the proportion correct is lower for fingers together compared to fingers spread $(t(9)=4.86$, $P<0.001)$ and lower for fingers interwoven compared to fingers spread $(t(9)=6.91, P<0.001)$. Fingers together and fingers interwoven are not significantly different $t(t)=1.99$, $P=0.07)$. Thus, increasing inter-finger distance indeed led to a more accurate localization of tactile stimuli.

\section{Mislocalization}

In the last paragraph of the introduction, we hypothesized that if the fingers are represented spatially, the error rates will be distributed differently for each finger configuration.
More specifically, the additional errors in the "fingers together" condition compared to the "fingers spread" condition should consist mainly of selecting the neighbouring dot on the neighbouring finger of the same hand, because that is the distance that changes most dramatically when the hand's configuration changes. Besides determining the proportion of responses that was correct, we therefore also determined the proportion of responses for specific response options (see Table 1): (1) the proportion of responses that was shifted by one position within a fingertip when the middle dot was stimulated, (2) the proportion of responses that was shifted by one position within a fingertip when one of the side dots was stimulated, (3) the proportion of responses that was shifted by one position to the neighbouring fingertip (which can obviously only happen when one of the side dots was stimulated), (4) the proportion of responses that was shifted by two positions within a fingertip, (5) the proportion of responses that was shifted by two positions to a neighbouring fingertip and (6) the proportion of shifts of more than two positions. We defined a shift to the neighbouring fingertip in spatial terms, which is to a fingertip of the other hand in the interwoven condition

Table 1 Values and correction factors that were used for the filled and open bars of Fig. 2

\begin{tabular}{|c|c|c|c|c|c|c|c|}
\hline $\begin{array}{l}\text { Distance } \\
\text { (dots) }\end{array}$ & Response & $\begin{array}{l}\text { Hand } \\
\text { configuration }\end{array}$ & $\begin{array}{l}\text { Proportion } \\
\text { of trials } \\
\text { (uncorrected) }\end{array}$ & $\begin{array}{l}\text { Number of possible } \\
\text { responses for each } \\
\text { relevant trial }\end{array}$ & $\begin{array}{l}\text { Proportion } \\
\text { of relevant } \\
\text { trials }\end{array}$ & $\begin{array}{l}\text { Correction } \\
\text { factor }\end{array}$ & $\begin{array}{l}\text { Normalized } \\
\text { value }\end{array}$ \\
\hline \multirow[t]{3}{*}{0} & \multirow[t]{3}{*}{ Correct } & Together & 0.443 & 1 & $150 / 150$ & 1 & 0.443 \\
\hline & & Spread & 0.557 & & & & 0.557 \\
\hline & & Interwoven & 0.402 & & & & 0.402 \\
\hline \multirow[t]{3}{*}{1} & \multirow[t]{3}{*}{ Same finger (from middle dot) } & Together & 0.101 & 2 & $50 / 150$ & 1.5 & 0.152 \\
\hline & & Spread & 0.093 & & & & 0.140 \\
\hline & & Interwoven & 0.087 & & & & 0.130 \\
\hline \multirow[t]{3}{*}{1} & \multirow[t]{3}{*}{ Same finger (from side dot) } & Together & 0.101 & 1 & $100 / 150$ & 1.5 & 0.151 \\
\hline & & Spread & 0.108 & & & & 0.162 \\
\hline & & Interwoven & 0.121 & & & & 0.181 \\
\hline \multirow[t]{3}{*}{1} & \multirow[t]{3}{*}{ Different finger (from side dot) } & Together & 0.014 & 1 & $100 / 150$ & 1.5 & 0.021 \\
\hline & & Spread & 0.003 & & & & 0.005 \\
\hline & & Interwoven & 0.013 & & & & 0.020 \\
\hline \multirow[t]{3}{*}{2} & \multirow[t]{3}{*}{ Same finger (from side dot) } & Together & 0.016 & 1 & $100 / 150$ & 1.5 & 0.024 \\
\hline & & Spread & 0.018 & & & & 0.028 \\
\hline & & Interwoven & 0.019 & & & & 0.029 \\
\hline \multirow[t]{3}{*}{2} & \multirow[t]{3}{*}{ Different finger (from side dot) } & Together & 0.011 & 1 & $100 / 150$ & 1.5 & 0.016 \\
\hline & & Spread & 0.009 & & & & 0.014 \\
\hline & & Interwoven & 0.014 & & & & 0.021 \\
\hline \multirow[t]{3}{*}{2} & \multirow[t]{3}{*}{ Different finger (from middle dot) } & Together & 0.012 & 2 & $50 / 150$ & 1.5 & 0.018 \\
\hline & & Spread & 0.008 & & & & 0.012 \\
\hline & & Interwoven & 0.010 & & & & 0.016 \\
\hline \multirow[t]{3}{*}{$>2$} & \multirow[t]{3}{*}{ Different finger } & Together & 0.302 & 25 & $150 / 150$ & 0.04 & 0.012 \\
\hline & & Spread & 0.203 & & & & 0.008 \\
\hline & & Interwoven & 0.333 & & & & 0.013 \\
\hline
\end{tabular}


Fig. 2 Proportion of responses in experiment 1 averaged over all participants (with standard errors). a Symbols (and open bars) proportions of responses. Solid bars proportions normalized by the number of possibilities of this response occurring (for details, see text; values are given in Table 1). Inset the critical values of the normalized responses, enlarged for clarity. b Normalized proportions for the distance in numbers of fingers from the target on the stimulated hand (upper panel) or the other hand (lower panel). The positions on the other hand are calculated as if the same finger of that hand was stimulated (e.g. if the middle finger was stimulated, an error of distance 1 would be to either the index or the ring finger. An error of distance 1 on the other hand would be to the index or ring finger of the other hand)
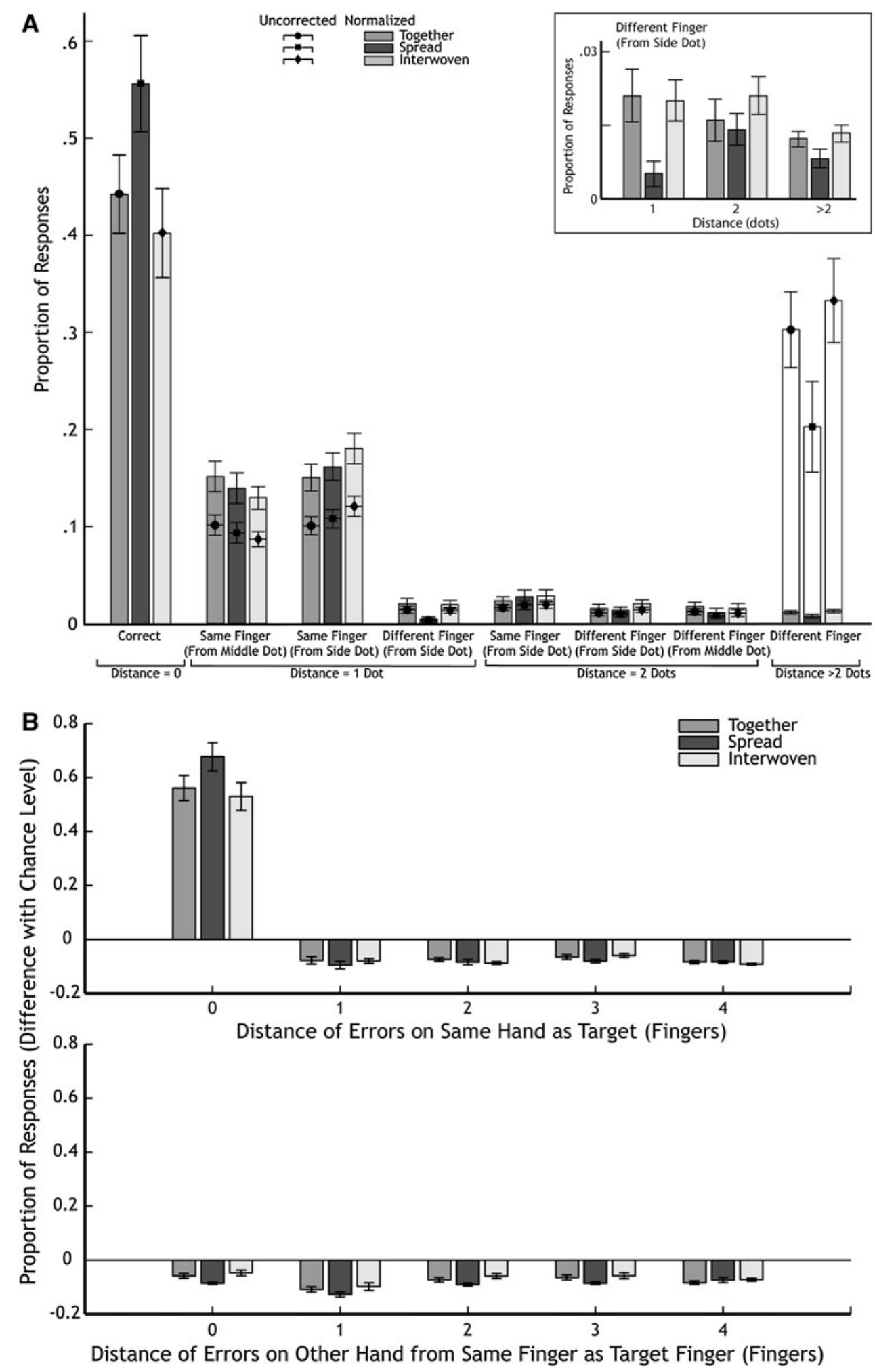

(below we will examine these data in somatotopic terms; Fig. 2b). The proportions of responses are plotted in Fig. 2a (transparent bars with symbols) and shown in the fourth column of Table 1. Because we considered all possible answers (correct and errors), these values add up to 1 for each finger configuration.

In order to check whether the adjacent position on the neighbouring fingertip is chosen exceptionally often, we 
normalized all the frequencies of responses. We did this by taking the number of possibilities to make such a response into account. Moreover, we also normalized for the number of trials in which certain kinds of dots were stimulated. For responses on the same fingertip, when the middle dot was stimulated, we divided the proportion of responses by two, because there are two possible responses that fall within this category (indicating the position to the left or right of the dot in question). However, the middle dot was only stimulated in one third of the trials, so we multiply the proportion of responses by 3 . The resulting correction factor (with respect to correct responses for which there was exactly one possibility on each trial) was therefore 1.5. We corrected every distance category according to the same principle. The corrected values are shown in Fig. 2a, and these values and the normalization factors can be found in Table 1.

We performed a repeated-measures ANOVA with the factors 'distance category' (the eight categories shown in the Table 1 and Fig. 2) and 'hand configuration' on these data: we found a main effect for distance category $(F(7.63)=76.83, P<0.001)$, a main effect for hand configuration $(F(2.18)=14.26, P<0.001)$ and an interaction effect between the two factors $(F(14.126)=12.16$, $P<0.001)$. Figure 2 a clearly shows that a neighbouring dot on the stimulated fingertip ("same finger") is chosen relatively frequently. The probability of choosing the neighbouring dot on the next fingertip was not clearly higher than that of choosing any other dot on another fingertip (in any condition). When one of the side dots was stimulated, the probability of choosing a neighbouring dot within the same fingertip was much higher than choosing the neighbouring dot on the neighbouring fingertip (same finger-side dot vs. different finger-side dot).

To check our specific prediction that spreading the fingers would decrease the error rate to the neighbouring fingertip (compared to 'fingers together'), we performed a separate repeated-measures ANOVA on all distance categories that are to a different fingertip (distance 1 from side dot, distance 2 from side dot, distance 2 from middle dot and anywhere else, see inset in Fig. 2a). We found a main effect of finger configuration $(F(2.18)=5.76, P<0.05)$ but no effect for distance category $(F(3.27)=2.17, P=0.12)$ and no interaction effect $(F(6.54)=1.20, P=0.32)$, indicating that each distance category is chosen equally often. Although fewer errors were made whereby a position at a 1-dot distance on the adjacent fingertip was chosen in the fingers spread condition, this does not really support the idea that spreading the fingers reduces the error rate by increasing the distances between the (potential) stimulation points, because the value for the 1-dot difference is exceptionally low. If it were just a matter of distance, then the value would decrease to that of the 2-dot or larger differ- ences, but, in fact, it appears to be even smaller than those. Since this was not significant (neither the interaction nor the distance category), the lower error rate in this particular case is considered to be a coincidence. Thus, the main (and consistent) difference between the conditions is a lower overall error rate in the fingers spread condition.

To test the hypothesis of Haggard et al. (2006) that the hands are localized separately from the fingers, we determined whether the identity of the hand matters in localizing the stimulus. We plotted the (somatotopic) distance of the response from the target finger in terms of the number of fingers rather than positions on the fingers (Fig. 2b), as was already mentioned above. The distance from the stimulated fingertip to a selected fingertip within the target hand is shown in the upper panel, and the distance from the corresponding fingertip of the other hand is shown in the lower panel. To obtain a comparable measure for all distances despite the different number of possibilities, we normalized the responses: the bars shown in Fig. $2 b$ are the difference between the proportion of responses made and the proportion of responses that we would expect to have been made if participants were randomly guessing (chance level: when guessing, the chance that you guess any of the fingers is 0.1 ; e.g. when the middle finger is stimulated, the chance of giving a response that is distance 2 on the same hand that was stimulated is 0.2: 0.1 for the little finger and 0.1 for the thumb, which are the two fingers that are distance 2 away from the middle finger; when stimulating the ring finger, the chance of responding with distance 2 is only 0.1 : the only possibility here is the index finger; for responding correctly, the chance level is always 0.1 ). To test whether the normalized responses were evenly distributed across the fingertips, we performed a 2-factor repeated-measures ANOVA on the number of incorrect responses ( 9 distances, excluding distance 0 on the same hand, which are correct responses; 3 finger configurations). We found a main effect for configuration $(F(2.18)=14.77, P<0.001)$, a main effect for distance $(F(8.72)=11.78, P<0.001)$ and an interaction between distance and configuration $(F(16.144)=2.26, \quad P<0.01)$. We performed post hoc paired-samples $t$ tests (Bonferroni-corrected) to investigate the causes of the main effects and interaction. For the main effect of configuration, we found that both fingers together and fingers interwoven have significantly more incorrect responses compared to fingers spread $\left(t_{d f=89}=3.19\right.$, $P<0.01$, and $t_{d f=89}=4.36, P<0.0001$, respectively). For the main effect of distance, we found that distance 1 on the other hand is significantly lower compared to distance 2 and 3 on the same hand and distance $0,2,3$ and 4 of the other hand $\quad\left(t_{d f=29}=5.03, \quad t_{d f=29}=8.14, \quad t_{d f=29}=9.00\right.$, $t_{d f=29}=6.80, t_{d f=29}=7.84$ and $t_{d f=29}=6.02$, respectively; all $P<0.0001)$. For the interaction effect, we performed a post hoc paired-samples $t$ test (Bonferroni-corrected) on all 
distance categories for each configuration, and the only significant difference was between distance 0 and 1 on the other hand for 'fingers together' $\left(t_{d f=9}=7.30, P<0.001\right)$, between distance 1 on the other hand and distance 2, 3 and 4 on the same hand and distance $0,2,3$ and 4 on the other hand for 'fingers spread' $\left(t_{d f=9}=8.89, t_{d f=9}=7.26, t_{d f=9}=\right.$ $6.33, t_{d f=9}=6.09, t_{d f=9}=-6.08, t_{d f=9}=-9.17$, and $t_{d f=9}=$ -6.05, respectively; all $P<0.001$ ), and between distance 1 on same hand and distance 3 on the other hand for 'fingers interwoven' $\left(t_{d f=9}=-5.48, P<0.001\right)$. Taken together, except for the effects of hand configuration, no clear pattern of results arises from these post hoc tests.

If Haggard's hypothesis were correct, we should have found a significant increase in responses to the same fingertip as the stimulated fingertip but situated on the opposite hand (distance 0 in lower panel of Fig. 2b). Although we found a main effect for distance, this effect was not caused by an increase in responses to the same fingertip on the other hand.

\section{Experiment 2}

In experiment 1 , we found a general reduction in error rate in the fingers spread condition compared to the fingers together and fingers interwoven conditions. This may be explained by the fingers touching each other in the fingers together and fingers interwoven conditions. When the fingers are touching each other, additional tactile input is given to the fingers. If tactile perception adapts to this constant input, the detection threshold for tactile input could become higher. A higher threshold will in turn result in more randomly distributed errors, which is the result that we found in experiment 1 . To test this hypothesis, we designed experiment 2 , in which we keep the finger position stable across conditions, but we vary the tactile input at the sides of the fingers.

\section{Method}

\section{Participants}

Ten participants, five men and five women with an average age of 26.5 years (range 23-29) participated in this experiment. The participants were undergraduate students and co-workers. None of the participants had problems with tactile perception, due to, for example, numbness, callus or scar tissue on any of their fingers. We did not consider handedness to be an important factor in the current study. All participants signed an informed consent before participating in the experiment. The study was part of a programme that was approved by the ethical committee of the Faculty of Human Movement Sciences of the
VU University. None of the participants had participated in experiment 1 .

\section{Stimuli, set-up \& procedure}

The same stimuli, set-up and procedure were used as in experiment 1, but now with two conditions: fingers spread (the same as in experiment 1) and fingers spread with additional tactile input. The latter was realized by placing triangular pieces of foam between the participant's fingers so that the sides of the fingers had additional tactile input similar to the fingers together and fingers interwoven conditions in experiment 1 (see inset in Fig. 3). Half of the subjects started with fingers spread and the other half with fingers spread with additional tactile input.

\section{Results}

The mean error rate was $0.61 \pm .05$ in the fingers spread condition and $0.59 \pm .06$ in the fingers spread with additional tactile input condition (not significantly different from each other; paired-samples $t_{9}=.63, P=.54$; Fig. 3a). These values are similar to the value we found in experiment 1 for the fingers spread condition $(0.56 \pm 0.05)$. We performed a repeated-measures ANOVA with the factors finger configuration and distance category (as we did in experiment 1). We only found a main effect for distance category $\left(F_{7.63}=88.61, P<.001\right)$. We did not find a main effect for finger configuration or an interaction between the two factors $\left(F_{1,9}=1.43, P=.26\right.$ and $F_{7,63}=2.11, P=0.06$, respectively).

We also calculated the error rate at the level of the finger (i.e. errors within fingertips were counted as correct; Fig. 3b). The mean error rates were $.66 \pm .05$ and $.68 \pm .06$ for fingers spread and fingers spread with additional tactile input, respectively. We performed a repeated-measures ANOVA on the factors finger configuration and distance of the error (in number of fingers). We found a main effect for distance $\left(F_{8.72}=9.15, P<.001\right)$, but again no main effect for finger configuration and no interaction $\left(F_{1,9}=.35\right.$, $P=.57$ and $F_{8,72}=.93, P=0.50$, respectively).

The results of experiment 2 show that additional input on the sides of the fingers does not influence the results of a tactile localization task. Therefore, our current results cannot simply be explained by the amount of contact between the fingers in the different conditions.

\section{General discussion}

In the current study, we examined the effect of finger configuration on a tactile localization task. Our hypothesis was that there would be a lower error rate to the neighbouring 
Fig. 3 Results of experiment 2 in the same format as Fig. 2. The inset in a illustrates the two conditions. "Spread +" is the condition with additional tactile input
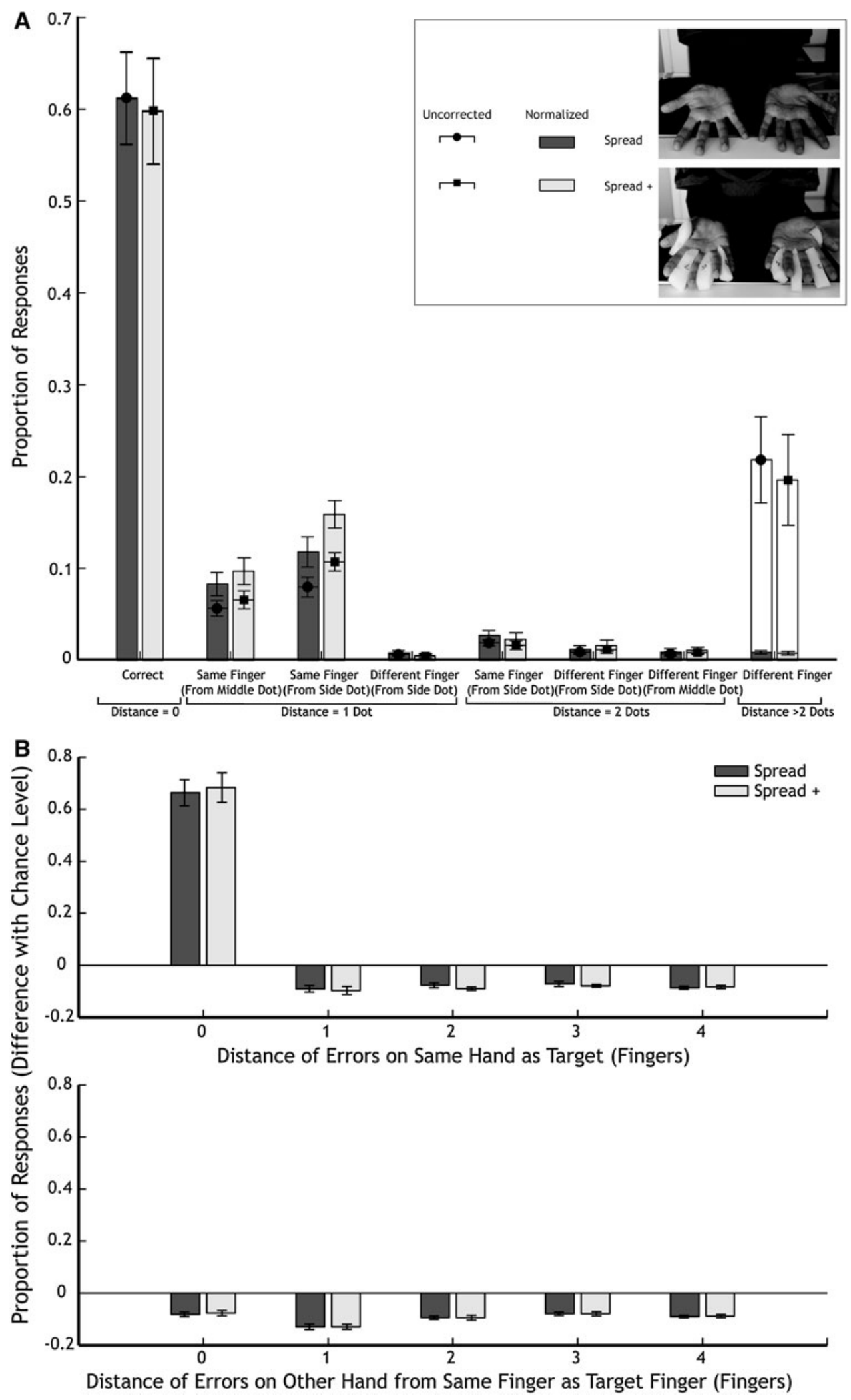

fingertip in the fingers spread configuration than in the other two configurations, because in the latter cases, one would have a larger spatial separation between the finger- tips. We indeed found a lower error rate in the "fingers spread' configuration compared to the 'fingers together' and 'fingers interwoven' configurations. However, in all 
three conditions, there were many more errors to the neighbouring dot on the same fingertip than to the neighbouring dot on the next fingertip (Fig. 2a). Moreover, overall, errors to the neighbouring fingertip occurred as frequently as errors to any other fingertip (Fig. 2b). Both at the dot level and at the finger level, the main difference between the conditions is the likelihood of correctly identifying the target. This indicates that spreading the fingers helps in localizing the target to a particular location on a fingertip but does not decrease the error rate of localization of stimuli onto the adjacent fingertip compared to the other fingertips. The improvement in localizing the target in the fingers spread condition is a global effect rather than being limited to avoiding a specific kind of errors.

It is quite surprising that we did not find more responses to neighbouring fingertips, as Schweizer et al. (2000) observed. Participants in the current study had to choose between thirty possible response options, distributed over the upper segments of the fingers of both hands. The participants in the study of Schweizer et al. had to choose between 42 response options, which were distributed over all segments of the fingers of one hand. It is possible that the spatial receptive fields that were used to detect our stimulus do not overlap across the fingers, but equivalent receptive fields on the lower segments of the fingers do overlap. The absolute distance between the lower segments of the fingers when they are spread is smaller than the distance between the upper segments. It is known that there are more afferents with small receptive fields than with large receptive fields at the fingertips. On the middle and lower segments of the finger, there are more large receptive fields (Vallbo and Johansson 1984). Unfortunately, Schweizer et al. did not specifically compare the error distributions for each finger segment separately, so whether this is responsible for the difference between our study and theirs needs further investigation.

There is neurophysiological evidence that there are overlapping finger representations in SI (e.g. Iwamura et al. 1983a, b; McKenna et al. 1982). A study by Fitzgerald et al. (2006) found that most receptive fields in SII also have overlapping finger representations. They claim that these receptive fields probably serve a more integrative function within the hands. Based on our finding that the error rate to the neighbouring fingertip was equal to that to the other fingertips, we hypothesize that these receptive fields in SI and SII are not used for fingertip identification.

Overall, fewer errors were made when the fingers were spread. In experiment 2, we showed that this is not due to additional tactile input from the contact between the fingers when they are 'together' or 'interwoven'. Moreover, most of the errors that are made in the current experiment are made within a finger, and the number of these errors does not differ between the different configurations (see Fig. 2a).
We found that the spatial configuration of the fingers influences the ability to distinguish between the fingertips, which implies that information from proprioception is taken into account when localizing a stimulus on the fingertips. This means that the location of the individual fingers in the external coordinate system is taken into account at some point during the processing of the tactile stimulus. This may be a representation in spatial coordinates, but the fingers' positions may also be represented relative to each other, in which case the range of possibilities that need to be considered would be limited because the fingers are always close to each other. These accounts could both explain why we found a general decrease in errors when spreading the fingers, instead of finding that the distribution of errors is different in the different configurations as we had hypothesized.

A recent study by Roberts and Humphreys (2010) shows that finger position influences perception of texture. Their participants had to indicate the roughness of a patch of sandpaper presented to one finger, while a distractor patch of sandpaper was presented to another finger. The influence of the distractor was modulated by the posture of the target and distractor fingers. This is in line with the current results, where we also found an influence of finger position on task performance.

Other studies found that interweaving the fingers reduces the accuracy of performance on certain tasks. A classical example is the Japanese illusion (Van Riper 1935): when we interleave our fingers, we make many errors in lifting the finger that is pointed at by someone else. In a study by Zampini et al. (2005), participants had to judge the order in which a pair of vibrotactile stimuli were presented to two adjacent fingers. When the participant's hands were placed side by side, directional discrimination performance was generally accurate. By contrast, when the fingers of the two hands were interleaved, with the fingers either pointing away from the body or else pointing towards the midline, performance deteriorated significantly for certain combinations of digits, with a more pronounced impairment when the fingers pointed away from the participant than when they pointed towards the midline. Röder et al. (2002) found no effect on tactile target detection when interleaving the fingers in the latter manner. One could argue that interweaving the fingers with the hands facing each other reduces the effect of interweaving because the direction of discrimination does not involve a left versus right discrimination. In our study, we found more errors in the interwoven condition compared to the fingers spread condition. The fingers were pointing away from the participant, and therefore, our results are consistent with earlier findings. Thus, the fact that we found a similar error rate in the fingers interwoven and fingers together conditions and a higher error rate in the fingers interwoven condition compared to 
the fingers spread condition indicates once more that a spatial explanation can account for the current results.

Haggard et al. (2006) claimed that the hands are localized separately from the fingers: identifying a finger does not immediately imply which hand it belongs to. If that were so, one would expect an exceptionally large number of responses to the same finger of the opposite hand. We did not find this for any of the three finger configurations (see Fig. 2b). This indicates that the fingers are not localized completely separately from the hands. The location of the fingers is connected to the location of the hand they belong to.

In summary, our results show that spreading the fingers improves localization of a tactile stimulus on the fingertip compared to holding the fingers together or interweaving them (experiment 1). This is not due to the additional contact at each finger (experiment 2), so the larger sensitivity when increasing the distance between the fingertips suggests a spatial representation. However, the results cannot simply be explained by receptive fields that extend across neighbouring fingers, because there is no specific tendency to name the neighbouring fingertip (compared to any other finger) in trials in which an error is made. Moreover, when spreading the fingers, there was no specific decrease in errors towards a neighbouring fingertip. The probability of missing the stimulation decreases when the fingers are spread, but the accuracy of localizing detected stimuli is similar in the three conditions. Tactile receptive fields with a spatial extent that depend on proprioception (i.e. configuration of the hand) may become more sensitive if less skin surface falls within the receptive field. That sensitivity of receptive fields can change in relation to stimulus strength has already been shown by Johansson (1976, 1978), but the current results suggest that the sensitivity of receptive fields can also depend on changes in proprioceptive input.

Acknowledgments This work was supported by Vidi grants from NWO (Netherlands Organization for Scientific Research, 452-03-325 to H. Chris Dijkerman and 452-02-073 to Jeroen B.J. Smeets). We want to thank Christa Nijnens for her help in collecting the data of experiment 2 .

Open Access This article is distributed under the terms of the Creative Commons Attribution Noncommercial License which permits any noncommercial use, distribution, and reproduction in any medium, provided the original author(s) and source are credited.

\section{References}

Azañon E, Soto-Faraco S (2008) Changing reference frames during the encoding of tactile events. Curr Biol 18(14):1044-1049

Craig JC (2003) The effect of hand position and pattern motion on temporal order judgments. Percept Psychophys 65(5):779-788
Fitzgerald PJ, Lane JW, Thakur PH, Hsiao SS (2006) Receptive field $(\mathrm{RF})$ properties of the macaque second somatosensory cortex: RF size, shape, and somatotopic organization. J Neurosci 26(24): 6485-6495

Haggard P, Kitadono K, Press C, Taylor-Clarke M (2006) The brain's fingers and hands. Exp Brain Res 172(1):94-102

Iwamura Y, Tanaka M, Sakamoto M, Hikosaka O (1983a) Converging patterns of finger representation and complex response properties of neurons in area 1 of the first somatosensory cortex of the conscious monkey. Exp Brain Res 51:327-337

Iwamura Y, Tanaka M, Sakamoto M, Hikosaka O (1983b) Functional subdivisions representing different finger regions in area 3 of the first somatosensory cortex of the conscious monkey. Exp Brain Res 51:315-326

Johansson RS (1976) Receptive-field sensitivity profile of mechanosensitive units innervating glabrous skin of human hand. Brain Res 104(2):330-334

Johansson RS (1978) Tactile sensibility in human hand-receptivefield characteristics of mechanoreceptive units in glabrous skin area. J Physiol (London) 281:101-123

Kaas JH (1983) What, if anything, is SI? Organization of first somatosensory area of cortex. Physiol Rev 63(1):206-231

McKenna TM, Whitsel BL, Dreyer DA (1982) Anterior parietal cortical topographic organization in macaque monkey: a reevaluation. J Neurophysiol 48(2):289-317

Natsoulas T, Dubanoski RA (1964) Inferring the locus and orientation of the perceiver from responses to stimulation of the skin. Am J Psychol 77:281-285

Overvliet KE, Mayer KM, Smeets JBJ, Brenner E (2008) Haptic search is more efficient when the stimulus can be interpreted as consisting of fewer items. Acta Psychol 127(1):51-56

Roberts RD, Humphreys GW (2010) The role of somatotopy and body posture in the integration of texture across the fingers. Psychol Sci 21(4):476-483

Röder B, Spence C, Rosler F (2002) Assessing the effect of posture change on tactile inhibition-of-return. Exp Brain Res 143(4):453462

Schicke T, Röder B (2006) Spatial remapping of touch: confusion of perceived stimulus order across hand and foot. Proc Natl Acad Sci USA 103(31):11808-11813

Schweizer R, Maier M, Braun C, Birbaumer N (2000) Distribution of mislocalizations of tactile stimuli on the fingers of the human hand. Somatosens Mot Res 17(4):309-316

Schweizer R, Braun C, Fromm C, Wilms A, Birbaumer N (2001) The distribution of mislocalizations across fingers demonstrates training-induced neuroplastic changes in somatosensory cortex. Exp Brain Res 139(4):435-442

Soto-Faraco S, Ronald A, Spence C (2004) Tactile selective attention and body posture: assessing the multisensory contributions of vision and proprioception. Percept Psychophys 66(7):1077-1094

Vallbo AB, Johansson RS (1984) Properties of cutaneous mechanoreceptors in the human hand related to touch sensation. Hum Neurobiol 3(1):3-14

Van Riper C (1935) An experimental study of the Japanese illusion. Am J Psychol 47:252-263

Weinstein S (1968) Intensive and extensive aspects of tactile sensitivity as a function of body part, sex, and laterality. In: Kenshalo DR (ed) The skin senses. Charles L. Thomas, Springfield, pp 195-222

Yamamoto S, Kitazawa S (2001) Reversal of subjective temporal order due to arm crossing. Nat Neurosci 4(7):759-765

Zampini M, Harris C, Spence C (2005) Effect of posture change on tactile perception: impaired direction discrimination performance with interleaved fingers. Exp Brain Res 166(3-4):498-508 